\title{
The moment problem for non-compact semialgebraic sets
}

\author{
Victoria Powers and Claus Scheiderer \\ (Communicated by the Managing Editors)
}

\section{Introduction and background}

Given a closed subset $K$ of $\mathbb{R}^{n}$, and $\left(m_{\alpha}\right)_{\alpha \in \mathbb{Z}_{+}^{n}}$ a (multi-) sequence of real numbers, the $K$-moment problem asks whether this sequence can be realized as the moment sequence of some positive Borel measure on $K$. In other words, the question is whether there is a positive Borel measure $\mu$ on $K$ which satisfies

$$
\int_{K} x^{\alpha} d \mu=m_{\alpha}
$$

for every $\alpha \in \mathbb{Z}_{+}^{n}$. (By this notation, we imply tacitly that all moments of $\mu$ exist.) In slightly different terms, the question is to characterize those linear forms $L: \mathbb{R}\left[t_{1}, \ldots, t_{n}\right] \rightarrow \mathbb{R}$ for which there exists a positive Borel measure $\mu$ on $K$ whose moments exist and satisfy

$$
\int_{K} f(x) d \mu=L(f)
$$

for every $f \in \mathbb{R}\left[t_{1}, \ldots, t_{n}\right]$. In this case, we say that the $K$-moment problem is solvable for $L$. Obviously, it is necessary that $L(f) \geqslant 0$ whenever $f \geqslant 0$ on $K$. A classical theorem says that this condition is also sufficient:

1.1. Theorem (Haviland [12]). The K-moment problem is solvable for L if and only if $L(f) \geqslant 0$ for every polynomial $f$ which is non-negative on $K$.

Moment problems were originally studied in the one-variable case. In 1894, Stieltjes [25] showed that for $K=[0, \infty)$, the $K$-moment problem is solvable for $L$ if and only if $L\left(f^{2}+t g^{2}\right) \geqslant 0$ for all $f, g \in \mathbb{R}[t]$. The most famous example is the case $K=\mathbb{R}$, which was solved by Hamburger in 1921 [9]. He showed that a necessary and sufficient condition in this case is $L\left(f^{2}\right) \geqslant 0$ for all $f \in \mathbb{R}[t]$. In 1923, Hausdorff [11] studied the case $K=[0,1]$ and showed that a necessary and sufficient condition is $L\left(f^{2}+t g^{2}+(1-t) h^{2}\right) \geqslant 0$ for all $f, g, h \in \mathbb{R}[t]$. These results can be viewed as par- 
ticular cases of Haviland's theorem; in all three cases, every polynomial which is nonnegative on $K$ is a finite sum of polynomials which were used for testing $L$.

Extending the moment problem to more than one variable is a more recent idea. The multidimensional moment problem is mentioned briefly in the 1943 book of Shohat and Tamarkin [24]. Some partial results on the two-dimensional moment problem are given in a paper by Devinatz [8] in 1957. In 1979, Schmüdgen [21] and Berg, Christensen, and Jensen [1] showed that the Hamburger result for $K=\mathbb{R}$ does not extend to $\mathbb{R}^{n}$ for $n \geqslant 2$, using the fact that in more than one variable there exist polynomials which are globally non-negative but not sums of squares (see below). Later works studied the multidimensional moment problem for other (specific) sets $K$, see, e.g., [2], [6], [16].

In the following, we write $A=\mathbb{R}\left[t_{1}, \ldots, t_{n}\right]$, and denote the set of sums of squares in $A$ by $\Sigma A^{2}$.

1.2. Definition. Given a closed subset $K$ of $\mathbb{R}^{n}$ and a subset $P$ of $A$, we say that $P$ solves the moment problem for $K$ if

(1) $K=\left\{x \in \mathbb{R}^{n}: f(x) \geqslant 0\right.$ for every $\left.f \in P\right\}$;

(2) for every linear functional $L$ on $A$ with

$$
L\left(a^{2} f_{1} \ldots f_{r}\right) \geqslant 0
$$

for every $a \in A, r \geqslant 0$ and $f_{1}, \ldots, f_{r} \in P$, there is a positive Borel measure $\mu$ on $K$ such that $L$ is integration with respect to $\mu$.

The classic examples above imply (for $n=1$ ) that $\{t\}$ solves the moment problem for $[0, \infty), \varnothing$ solves the moment problem for $\mathbb{R}$, and $\{t, 1-t\}$ solves the moment problem for $[0,1]$.

In 1991, K. Schmüdgen proved the following remarkable theorem:

1.3. Theorem (Schmüdgen [23]). Suppose $f_{1}, \ldots, f_{r} \in A$ are such that $K:=$ $\left\{f_{1} \geqslant 0, \ldots, f_{r} \geqslant 0\right\}$ is compact. Then $\left\{f_{1}, \ldots, f_{r}\right\}$ solves the moment problem for $K$.

Note that Schmüdgen's theorem holds regardless of the polynomials chosen to define $K$ by inequalities. As a simple example, we obtain the following (non-obvious) variation of Hausdorff's result: Let $K=[0,1]$, then for any (fixed) odd integers $k$ and $m$, the $K$-moment problem is solvable for $L$ if and only if $L\left(f^{2}+g^{2} t^{k}(1-t)^{m}\right) \geqslant 0$ for all $f, g \in \mathbb{R}[t]$.

It seems that Schmüdgen's result was the first on the moment problem which covers a truly general class of sets $K$, rather than just specific sets.

Our goal in this paper is to study the non-compact case. Recall that a subset $K$ of $\mathbb{R}^{n}$ is called basic closed (semialgebraic) if it has the form $K=\left\{f_{1} \geqslant 0, \ldots, f_{r} \geqslant 0\right\}$, where the $f_{i}$ are polynomials. Given such $f_{i}$, we ask, when does $\left\{f_{1}, \ldots, f_{r}\right\}$ solve the moment problem for $K$ ? The result of Berg et al. and Schmüdgen mentioned above shows that Schmüdgen's theorem does not generalize to the non-compact case: 
1.4. Example. Suppose $A=\mathbb{R}\left[t_{1}, \ldots, t_{n}\right]$ where $n \geqslant 2$. Then there exists a linear functional $L$ on $A$ such that $L\left(f^{2}\right) \geqslant 0$ for all $f \in A$ but the $\mathbb{R}^{n}$-moment problem is not solvable for $L$. In particular, $\varnothing$ does not solve the moment problem for $\mathbb{R}^{n}$.

Sketch of proof. Since $n \geqslant 2$, there exists a polynomial $p$ such that $p \geqslant 0$ on all of $\mathbb{R}^{n}$, but $p$ is not a sum of squares. This was proven by Hilbert in 1888 [13], although the first explicit example of such $p$ to appear in the literature was given by Motzkin in 1965 [17]. (See [18] for more on this interesting subject.)

The cone $\Sigma A^{2}$ is closed in the finest locally convex vector space topology on $A$. By Hahn-Banach separation of convex sets, there is a linear functional $L$ on $A$ such that $L(p)<0$ but $L(f) \geqslant 0$ for every $f \in \Sigma A^{2}$. Trivially, $L$ cannot come from a positive Borel measure on $\mathbb{R}^{n}$.

Note that Haviland's theorem implies immediately that the set $P$ consisting of all everywhere non-negative polynomials in $A$ solves the moment problem for $\mathbb{R}^{n}$. More generally, for any closed $K$, there exists a set $P$ which solves the moment problem for $K$, namely the set of all $f$ which are non-negative on $K$. However, even if $K$ is a basic closed semialgebraic set, it is not clear - and not true - in general that a finite set $P$ of polynomials can be found which solves the moment problem for $K$. Therefore, we wish to study the following questions:

- Given polynomials $f_{1}, \ldots, f_{r}$, can we give necessary or sufficient conditions under which they solve the moment problem for $K=\left\{f_{1} \geqslant 0, \ldots, f_{r} \geqslant 0\right\}$ ?

- Given a basic closed semialgebraic set $K$, when does there exist some finite set of polynomials which solves the moment problem for $K$ ?

If $K$ is compact, the answer to the second question is "always", and no conditions are needed in the first question, both by Schmüdgen's theorem. On the other hand, we will show that there are many cases of non-compact $K$ where the answer to the second question is negative.

If $P$ is any set of polynomials, it is easy to see that $P$ solves the moment problem for $K$ if and only if the closure of $P \cup \Sigma A^{2}$ under addition and multiplication does. A subset of $A$ closed under addition and multiplication and containing all squares is called a preorder. (See the next section for precise definitions.) It turns out to be advantageous to replace $P$ by the preorder it generates, and to study the moment problem for preorders. We will do so in the next section, after introducing the necessary technical language. Also, it will be important to allow arbitrary finitely generated $\mathbb{R}$ algebras $A$ in place of the polynomial ring. We equip $A$ with the finest locally convex vector space topology. The saturation of a preorder $P$ is the set of all polynomials which are non-negative on the closed set associated to $P$. By Haviland's theorem, $P$ solves the moment problem for its associated set if and only if the topological closure of $P$ is also closed under saturation (Cor. 3.1). For this reason we are interested in methods for deciding if a preorder is closed. A key notion in this regard is that of stable preorders. Our first main result is Theorem 2.14, which provides large classes of examples of basic closed sets $K$ for which every finitely generated preorder with associated set $K$ is topologically closed, but not saturated, and hence does not solve the moment problem for $K$. 
Thus we have found many cases where the answer to our second question above is negative. Among them are even (many) cases where $\operatorname{dim}(K)=1$ (Thm. 3.9). We also have positive results for 1-dimensional sets $K$, which we obtain by applying theorems from [20] (Thm. 3.12). Combining 3.9 and 3.12, we have obtained a complete solution for the moment problem on smooth algebraic curves. This is the second main result of our paper. Furthermore, in all cases, our results give more precise information than just whether the moment problem is solved or not.

When we were in the final stages of writing this paper, we found out from Murray Marshall and Salma Kuhlmann that they had been studying similar questions. Their approach and techniques are somewhat different from ours, for example, they work only in the polynomial ring. Their preprint [15] contains a list of open questions, and at the end of our paper we settle some of these.

Acknowledgements. A talk of K. Schmüdgen at the 60th birthday celebration of M. Marshall led the first author to consider the questions studied in this paper. She thanks Prof. Schmüdgen for his inspiring talk, Prof. Marshall for having a birthday, and Salma and Franz-Viktor Kuhlmann for organizing the birthday conference and inviting her. The authors also thank M. Marshall and S. Kuhlmann for bringing their work to their attention.

\section{Preorders}

Preorders are important objects in real algebra, which in some sense play a role in semialgebraic geometry that is comparable to the role played by ideals in algebraic geometry. In this section we study preorders with a view towards answering the questions on the moment problem raised in the previous section. However, our results are also of independent interest.

We need to work with finitely generated (f. g.) R-algebras, rather than with polynomial rings only. In geometric terms, this means working with affine algebraic $\mathbb{R}$ varieties rather than with $n$-dimensional affine space only. First recall the basic dictionary between such varieties and f. g. $\mathbb{R}$-algebras.

To any f. g. $\mathbb{R}$-algebra $A$ there corresponds an affine algebraic variety $V$ over $\mathbb{R}$. (We use the word "variety" in a broad sense, it does not imply irreducible or reduced.) The correspondence between $A$ and $V$ is expressed by writing $V=\operatorname{Spec} A$ or $A=$ $\mathbb{R}[V]$. The variety $V$ comes together with its set $V(\mathbb{R})$ of real points; by definition $V(\mathbb{R})=\operatorname{Hom}_{\mathbb{R}}(A, \mathbb{R})$, the set of $\mathbb{R}$-algebra homomorphisms from $A$ to $\mathbb{R}$. Given $M \in V(\mathbb{R})$, the corresponding homomorphism $A \rightarrow \mathbb{R}$ is written $f \mapsto f(M)$ and is thought of as evaluation of the elements of $A$ in $M$. Note that if $A=\mathbb{R}\left[t_{1}, \ldots, t_{n}\right]$, then $V=\mathbb{A}^{n}=$ affine $n$-space, and $V(\mathbb{R})$ is simply $\mathbb{R}^{n}$.

The set $V(\mathbb{R})$ comes with a natural topology, which has the sets $\{M \in V(\mathbb{R})$ : $f(M)>0\}(f \in A)$ as a subbasis of open sets. A subset of $V(\mathbb{R})$ is called semialgebraic if it is a finite boolean combination (unions, intersections, complements) of these sets.

Choosing a finite system $x_{1}, \ldots, x_{n}$ of generators of $A$ gives an epimorphism $\mathbb{R}\left[t_{1}, \ldots, t_{n}\right] \rightarrow A, t_{i} \mapsto x_{i}$, and correspondingly, an embedding of $V(\mathbb{R})$ into $\mathbb{R}^{n}$ as a Zariski closed subset. Thus, one can think of $V(\mathbb{R})$ as a (Zariski closed) alge- 
braic subset of some $\mathbb{R}^{n}$, if one wishes, but it is often preferable not to fix such an embedding.

Note that the full algebra $A$ cannot be retrieved from $V(\mathbb{R})$, if $V(\mathbb{R})$ is given as an algebraic subset of $\mathbb{R}^{n}$, say. For example, $V(\mathbb{R})$ may be empty without $A$ being trivial, e.g., for $A=\mathbb{R}[t] /\left(t^{2}+1\right)$. All one gets back from $V(\mathbb{R})$ is the quotient $\operatorname{ring} A / N$, where $N$ is the so-called real nilradical of $A$ (see $[3,4.1]$ ). To get a complete dictionary between algebras and affine varieties, it would be necessary to employ the structure sheaves of the latter.

Therefore, the algebra $A$ is our basic object of study. We fix $V=\operatorname{Spec} A$, an affine $\mathbb{R}$-variety. By this we mean that $A$ is a $\mathrm{f}$. g. $\mathbb{R}$-algebra and $V=\operatorname{Spec} A$ is the associated affine algebraic $\mathbb{R}$-variety. A subset $P$ of $A$ is called a preorder (in $A$ ) if $f^{2} \in P$ for every $f \in A$ and $P$ is closed under addition and multiplication. Any intersection of preorders in $A$ is again a preorder. Therefore, given a subset $F$ of $A$, there is a smallest preorder containing $F$, denoted $\mathrm{PO}(F)$, or $\mathrm{PO}\left(f_{1}, \ldots, f_{r}\right)$ if $F=\left\{f_{1}, \ldots, f_{r}\right\}$, and called the preorder generated by $F$. Explicitly, $\mathrm{PO}(F)$ is the set of all finite sums of elements of the form $a^{2} f_{1} \ldots f_{r}$, where $a \in A, r \geqslant 0$ and $f_{1}, \ldots, f_{r} \in F$.

Given a preorder $P$ in $A$, we write

$$
\mathscr{S}(P):=\{M \in V(\mathbb{R}): f(M) \geqslant 0 \text { for every } f \in P\} .
$$

This is a closed subset of $V(\mathbb{R})$. If $P=\mathrm{PO}\left(f_{1}, \ldots, f_{r}\right)$ is finitely generated, then $\mathscr{S}(P)=\left\{M \in V(\mathbb{R}): f_{1}(M) \geqslant 0, \ldots, f_{r}(M) \geqslant 0\right\}$, and in particular, is a semialgebraic set.

On the other hand, if $K$ is a closed subset of $V(\mathbb{R})$, we write

$$
\mathscr{P}(K):=\{f \in A: f \geqslant 0 \text { on } K\} .
$$

This is a preorder in $A$, and clearly $\mathscr{S}(\mathscr{P}(K))=K$.

A preorder $P$ will be called saturated if there exists a closed subset $K$ of $V(\mathbb{R})$ with $P=\mathscr{P}(K)$. If so, then necessarily $K=\mathscr{S}(P)$. The saturation $\operatorname{Sat}(P)$ of a preorder $P$ is defined by $\operatorname{Sat}(P):=\mathscr{P}(\mathscr{S}(P))$; this is the smallest saturated preorder containing $P$. The correspondence $K \mapsto \mathscr{P}(K)$ is a bijection between closed subsets of $V(\mathbb{R})$ and saturated preorders in $A$, the inverse map being $P \mapsto \mathscr{S}(P)$.

2.1. Examples. The unique smallest preorder in $A$ is $\Sigma A^{2}$, the set of sums of squares in $A$. Its saturation $\operatorname{Sat}\left(\Sigma A^{2}\right)$ is the set $A_{+}$of all positive semidefinite ( $\left.p s d\right)$ functions in $A$, i.e., of all $f \in A$ with $f \geqslant 0$ on $V(\mathbb{R})$. Consider in particular the case $A=$ $\mathbb{R}\left[t_{1}, \ldots, t_{n}\right]$. If $n=1$, then $\Sigma A^{2}=A_{+}$. However, if $n \geqslant 2$, then as we saw in Example $1.4, A_{+}$is strictly larger than $\Sigma A^{2}$.

As a matter of fact, the preorder $A_{+}$of all psd polynomials is not finitely generated (as a preorder), if $A=\mathbb{R}\left[t_{1}, \ldots, t_{n}\right]$ and $n \geqslant 2$. An even stronger assertion is true: $A_{+}$is not the closure of any finitely generated preorder, with respect to the topology introduced below. This is a particular case of Thm. 3.7 from the next section.

Let $A$ be any f. g. $\mathbb{R}$-algebra. By a subspace of $A$, we always mean an $\mathbb{R}$-linear subspace of $A$. Recall that if $W$ is any finite-dimensional (f.-d.) vector space over $\mathbb{R}$, then a semialgebraic set in $W$ is a finite boolean combination of sets of the form $\{x \in W: f(x)>0\}$, where $f$ is a polynomial function on $W$. A subset $S$ of $A$ will be 
called locally semialgebraic if $S \cap U$ is a semialgebraic subset of $U$ for every f.-d. subspace $U$ of $A$. If, in addition, $S$ is contained in some f.-d. subspace of $A$, then $S$ is called semialgebraic.

We will always equip $A$ with the finest topology that makes $A$ a locally convex topological $\mathbb{R}$-vector space [4, II $\S 4$ no. 2]. Every subspace of $A$ is closed, and every linear map $A \rightarrow \mathbb{R}$ is continuous (loc. cit., exercise 6). By $A^{\vee}$ we denote the dual of $A$, i.e., the space of all linear maps $A \rightarrow \mathbb{R}$. A subset of $A$ is closed if and only if its intersection with every f.-d. subspace $U$ of $A$ is closed in $U$. (This uses that $A$ has a countable linear basis, see loc. cit., exercise 8.)

For preorders in $A$, we study the properties of being closed and of being saturated. These will be key properties needed for our results on the moment problem. We begin with some simple observations.

2.2. Lemma. Any preorder in A is a convex cone in A. Any saturated preorder is closed in $A$.

Proof. The first statement is obvious. For the second, note that if $P=\mathscr{P}(K)$ is saturated, then $P=\bigcap_{M \in K} \lambda_{M}^{-1}[0, \infty)$, where $\lambda_{M}: A \rightarrow \mathbb{R}$ is evaluation at $M$.

By the Hahn-Banach separation theorem for convex sets [4, II $\S 5$ no. 3], the closure $\bar{P}$ of the preorder $P$ is given by

$$
\bar{P}=\left\{a \in A: L(a) \geqslant 0 \text { for every } L \in A^{\vee} \text { with } L \geqslant 0 \text { on } P\right\} .
$$

However, in practice it is often not easy to give a more concrete description of $\bar{P}$. By Haviland's theorem, the problem of understanding $\bar{P}$ is in fact closely related to the moment problem, see 3.1 below. It is precisely this relation which leads us to introduce the topology on $A$ and to study the closure $\bar{P}$ of a preorder $P$.

2.3. Lemma. If $P$ is a preorder in $A$, then the closure $\bar{P}$ of $P$ in $A$ is again a preorder. Moreover, $\mathscr{S}(P)=\mathscr{S}(\bar{P})$.

Proof. The multiplication map $A \times A \rightarrow A$ is continuous, since $A$ has a countable linear basis $[4$, II exercise 9a]. Therefore, it is clear that $\bar{P}$ is a preorder. Moreover, the saturated preorder $\operatorname{Sat}(P)$ is closed, and so $P \subset \bar{P} \subset \operatorname{Sat}(P)$, which implies $\mathscr{S}(P) \supset$ $\mathscr{S}(\bar{P}) \supset \mathscr{S}($ Sat $P)=\mathscr{S}(P)$.

2.4. Proposition. Let $K$ be a closed semialgebraic set in $V(\mathbb{R})$, and let $P=\mathscr{P}(K)$, the saturated preorder associated to $K$. Then $P$ is a closed, convex, locally semialgebraic subset of $A$.

Proof. We already know that $P$ is closed and convex. There is an epimorphism $\pi: \mathbb{R}\left[t_{1}, \ldots, t_{n}\right] \rightarrow A$, for some $n$, inducing an embedding $V(\mathbb{R}) \hookrightarrow \mathbb{R}^{n}$. Given a f.-d. subspace $U$ of $A$, choose a f.-d. subspace $U^{\prime}$ of $\mathbb{R}\left[t_{1}, \ldots, t_{n}\right]$ so that $\pi\left(U^{\prime}\right)=U$. Then $U^{\prime} \cap \pi^{-1}(P)$ consists of all $f \in U^{\prime}$ which are non-negative on the closed semialgebraic subset $K$ of $V(\mathbb{R}) \subset \mathbb{R}^{n}$. So $U^{\prime} \cap \pi^{-1}(P)$ is a semialgebraic subset of $U^{\prime}$, since it can be described by a formula in the first order language of ordered fields. 
2.5. Proposition. Let $U$ be a $f$.-d. subspace of $A$. Then there is an integer $p_{U}$ such that every sum of squares of elements of $U$ is a sum of $p_{U}$ squares of elements of $U$.

Proof. By $[7,4.2]$ this is true for $A=\mathbb{R}\left[t_{1}, \ldots, t_{n}\right]$. It is easy to see that this particular case implies the general one: Again use $\pi: \mathbb{R}\left[t_{1}, \ldots, t_{n}\right] \rightarrow A$, and lift $U$ to a f.-d. subspace $U^{\prime}$ of $\mathbb{R}\left[t_{1}, \ldots, t_{n}\right]$, as in the proof of Prop. 2.4. The result for $U^{\prime}$ then implies the result for $U$.

Given a subspace $W$ of $A$ and $f_{1}, \ldots, f_{r} \in A$, we denote by $\Sigma\left(W ; f_{1}, \ldots, f_{r}\right)$ the set of all sums

$$
\sum_{i \in\{0,1\}^{r}} s_{i} \cdot f_{1}^{i_{1}} \ldots f_{r}^{i_{r}}
$$

in which the $s_{i}$ are sums of squares of elements of $W$. Recall that the ring $A$ is said to be reduced if $a^{2}=0$ implies $a=0$, for every $a \in A$.

2.6. Proposition. Let $f_{1}, \ldots, f_{r} \in A$, and let $K=\mathscr{S}\left(f_{1}, \ldots, f_{r}\right)$. Let $W$ be a $f$-d. subspace of $A$. Then

(a) $\Sigma\left(W ; f_{1}, \ldots, f_{r}\right)$ is a convex semialgebraic subset of $A$.

(b) If $A$ is reduced and $K$ is Zariski dense in $V$, then $\Sigma\left(W ; f_{1}, \ldots, f_{r}\right)$ is closed.

Proof. We abbreviate $\Sigma\left(W ; f_{1}, \ldots, f_{r}\right)$ by $\Sigma(W)$. It is clear that $\Sigma(W)$ is contained in some f.-d. subspace $U$ of $A$, and that it is a convex set. Let $N=p_{W}$, see Proposition 2.5.

For $a \in A$, let $\operatorname{Ann}(a)=\{b \in A: a b=0\}$, the annihilator of $a$. This is an ideal of $A$, and the ring $A / \operatorname{Ann}(a)$ is reduced if $A$ is reduced. For $i \in\{0,1\}^{r}$, write $f^{i}:=f_{1}^{i_{1}} \ldots f_{r}^{i_{r}}$. Consider the map

$$
\phi: \bigoplus_{i \in\{0,1\}^{r}}\left(W / W \cap \operatorname{Ann}\left(f^{i}\right)\right)^{N} \rightarrow A
$$

defined by

$$
w=\left(\bar{w}_{i j}\right)_{i \in\{0,1\}^{r}, j=1, \ldots, N} \mapsto \phi(w)=\sum_{i \in\{0,1\}^{r}}\left(\sum_{j} w_{i j}^{2}\right) \cdot f^{i}
$$

Here $\bar{w}_{i j}=w_{i j}+\left(W \cap \operatorname{Ann}\left(f^{i}\right)\right)$; note that the right hand side is well-defined.

The map $\phi$ is a homogeneous quadratic polynomial map, and $\Sigma(W)$ is its image set. In particular, it is clear that $\Sigma(W)$ is a semialgebraic set. Now assume that the conditions of $(\mathrm{b})$ hold. We first show $\phi^{-1}(0)=\{0\}$. So let $w=\left(\bar{w}_{i j}\right)$ be a tuple with $\phi(w)=0$. If $M$ is any point in $K$, we have $f^{i}(M) \geqslant 0$ for every $i$, and therefore $\left(w_{i j}^{2} f^{i}\right)(M)=0$ for every bi-index $(i, j)$. Since $K$ is Zariski dense and $A$ is reduced, it 
follows that $w_{i j}^{2} f^{i}=0$, and hence even $w_{i j} f^{i}=0$, for every bi-index $(i, j)$. Hence $w=0$.

Now the next lemma shows that $\phi$ is a proper map. In particular, the image of $\phi$ is closed.

2.7. Lemma. Suppose $\phi=\left(\phi_{1}, \ldots, \phi_{n}\right): \mathbb{R}^{m} \rightarrow \mathbb{R}^{n}$ is a homogeneous map of some fixed degree $d \geqslant 1$ (i.e., each component $\phi_{i}$ is homogeneous of degree $d$ ). If $\phi^{-1}(0)=$ $\{0\}$, then $\phi$ is a proper map.

Proof. Recall that a continuous map is proper iff it is closed and has compact fibres [5, ch. I $\S 10$ no. 2]. If $K$ is a closed subset of $\mathbb{R}^{m}$ with $0 \notin K$, then $0 \notin \overline{\phi(K)}$, since $0 \notin \phi(K)$ and $\phi$ is homogeneous. Therefore, it suffices to show that the restriction $\phi^{\prime}: \mathbb{R}^{m} \backslash\{0\} \rightarrow \mathbb{R}^{n} \backslash\{0\}$ of $\phi$ is proper. Consider the commutative square

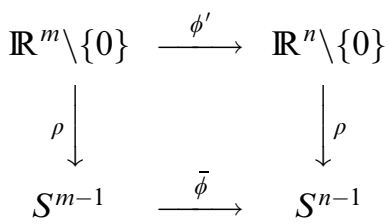

in which the vertical arrows are the natural retractions $x \mapsto x /|x|$ and $\bar{\phi}=\rho \circ \phi \mid S^{m-1}$. One checks immediately that the square $(*)$ is cartesian. Since $S^{m-1}$ is compact, $\bar{\phi}$ is a proper map, and therefore $\phi^{\prime}$ is proper as well.

2.8. Remark. In general, the conditions in Prop. 2.6(b) cannot be dropped. For example, let $A=\mathbb{R}[t], f=-t^{2}, K=\{0\}$ and $W=\mathbb{R} .1 \oplus \mathbb{R}$. $t$, the space of polynomials of degree $\leqslant 1$. Here $K$ is not Zariski dense in $V$. We have $t+\varepsilon \in \Sigma(W ; f)$ for every $\varepsilon>0$, but $t \notin \Sigma(W ; f)$, so $\Sigma(W ; f)$ is not closed. For essentially the same example, seen from a different viewpoint, take the finite (non-reduced) algebra $A=$ $\mathbb{R}[t] /\left(t^{2}\right)$ and $r=0\left(\right.$ no $\left.f_{i}\right)$. Here $t+\varepsilon$ is a sum of squares in $A$ for every $\varepsilon>0$, but not for $\varepsilon=0$. Again, $\Sigma A^{2}=\Sigma(A ; \varnothing)$ is not closed.

Let again $A$ be an arbitrary f. g. $\mathbb{R}$-algebra, and assume now that we are given finitely many elements $f_{1}, \ldots, f_{r} \in A$. Suppose we know that $\Sigma\left(W ; f_{1}, \ldots, f_{r}\right)$ is closed for every f.-d. subspace $W$ of $A$. Under suitable conditions, see 2.6(b), this will be the case. Let $P=\mathrm{PO}\left(f_{1}, \ldots, f_{r}\right)$, the preorder generated by the $f_{i}$. We would like to find a condition under which we can conclude that $P$ is itself closed. One such condition is the following:

(*) For every f.-d. subspace $U$ of $A$, there is a f.-d. subspace $W$ of $A$ with $P \cap U \subset$ $\Sigma\left(W ; f_{1}, \ldots, f_{r}\right)$.

Indeed, $P \cap U=\Sigma\left(W ; f_{1}, \ldots, f_{r}\right) \cap U$ then, which under our assumption is a closed subset of $U$.

We are therefore going to study condition $(*)$ more closely. 
2.9. Lemma. Let $f_{1}, \ldots, f_{r} \in A$, and write $P=\mathrm{PO}\left(f_{1}, \ldots, f_{r}\right)$. Given $g \in P$ (so also $\left.P=\mathrm{PO}\left(f_{1}, \ldots, f_{r}, g\right)\right)$, condition $(*)$ holds for $f_{1}, \ldots, f_{r}$ iff it holds for $f_{1}, \ldots, f_{r}, g$.

Proof. The "only if" is obvious. Conversely, assume $(*)$ holds for $f_{1}, \ldots, f_{r}, g$. Let $U$ and $W$ be f.-d. subspaces of $A$ with $U \cap P \subset \Sigma\left(W ; f_{1}, \ldots, f_{r}, g\right)$. Choose a f.-d. subspace $W^{\prime}$ of $A$ which contains 1 and the $f_{i}$ and in addition satisfies $g \in$ $\Sigma\left(W^{\prime} ; f_{1}, \ldots, f_{r}\right)$. Let $W W^{\prime}$ be the subspace spanned by the products $w w^{\prime}(w \in W$, $\left.w^{\prime} \in W^{\prime}\right)$. Then $U \cap P \subset \Sigma\left(W W^{\prime} ; f_{1}, \ldots, f_{r}\right)$.

2.10. Definition. Let $P=\operatorname{PO}\left(f_{1}, \ldots, f_{r}\right)$ be a finitely generated preorder in $A$. We say that $P$ is stable if $(*)$ holds. By Lemma 2.9 , this is independent of the choice of generators of $P$.

Note that if a $\mathrm{f}$. $\mathrm{g}$. preorder $P$ is stable, then it is a locally semialgebraic subset of $A$.

2.11. Corollary. Let $P$ be a finitely generated preorder in $A$ which is stable. Assume that $A$ is reduced and that $K=\mathscr{S}(P)$ is Zariski dense in $V$. Then $P$ is closed in $A$.

Proof. Choose $f_{1}, \ldots, f_{r} \in A$ with $P=\operatorname{PO}\left(f_{1}, \ldots, f_{r}\right)$. Then $\Sigma\left(W ; f_{1}, \ldots, f_{r}\right)$ is closed for each f.-d. subspace $W$ of $A$ (2.8). Since $P$ is stable, $P$ is closed.

2.12. Remark. Prof. Schmüdgen informs us that a condition similar to $(*)$ has been used in the context of general star algebras to prove that the positive cone is closed. See [22], condition (III) on page 326.

2.13. Remark. This remark is for readers with a little background in real algebraic geometry. It won't be used in the sequel.

Let $R$ be any real closed field, and let $A$ be a finitely generated $R$-algebra. Consider the topology on $A$ which is analogous to the one we use for $\mathbb{R}$-algebras: A subset of $A$ is open iff its intersection with every f.-d. $R$-linear subspace of $A$ is open in this subspace. Prop. 2.6 and Lemma 2.9 hold in this context as well, mutatis mutandis. Hence it is clear what we mean by saying that a finitely generated preorder in $A$ is stable.

Now one can give the following different characterizations of stable preorders, which explain the reason for our choice of the word "stable". Given a real closed field $R$, a finitely generated $R$-algebra $A$ and a finitely generated preorder $P$ in $A, P$ is stable if and only if either one of the following two conditions is satisfied:

(i) For every real closed field extension $R^{\prime} / R$, the preorder in $A^{\prime}=A \otimes_{R} R^{\prime}$ generated by $P$ is a locally semialgebraic subset of $A^{\prime}$;

(ii) $P$ is a locally semialgebraic subset of $A$, and for every real closed extension $R^{\prime}$ of $R$, the preorder in $A^{\prime}$ generated by $P$ is equal to $P\left(R^{\prime}\right)$.

Here in (ii), we mean by $P\left(R^{\prime}\right)$ the subset $Q$ of $A^{\prime}$ for which $Q \cap\left(U \otimes_{R} R^{\prime}\right)$ is the base extension (to $R^{\prime}$ ) of the $R$-semialgebraic set $P \cap U$, for every f.-d. $R$-linear subspace $U$ of $A$. 
We return to our usual setting, and assume that $A$ is a f. g. $\mathbb{R}$-algebra. The next theorem, though it may appear somewhat technical, is the first main result of our paper. In the next section we will apply it to concrete particular cases. It provides us with a large family of examples of finitely generated preorders which are stable and closed. On the other hand, if we add a suitable dimension hypothesis, they won't be saturated, by a result from [19].

Recall that $V=\operatorname{Spec} A$ is called normal if $A$ is a direct product of finitely many integrally closed domains. This is a mildness condition on the nature of the singularities of $A$.

2.14. Theorem. Suppose that the variety $V=\operatorname{Spec} A$ is normal. Let $P$ be a finitely generated preorder in $A$ and set $K=\mathscr{S}(P)$. Assume that $V$ has an open embedding into a normal complete $\mathbb{R}$-variety $\bar{V}$ such that the following is true: For any irreducible component $Z$ of $\bar{V}-V$, the subset $\bar{K} \cap Z(\mathbb{R})$ of $Z(\mathbb{R})$ is Zariski dense in $Z$, where $\bar{K}$ denotes the closure of $K$ in $\bar{V}(\mathbb{R})$. Then the preorder $P$ is stable and closed.

2.15. Example. To illustrate this, consider the polynomial $\operatorname{ring} A:=\mathbb{R}\left[t_{1}, \ldots, t_{n}\right]$ and the preorder $P=\Sigma A^{2}$ of all sums of squares in $A$. The preorder $P$ is stable (and therefore closed). Indeed, if a polynomial $f$ is a sum of squares, say $f=\sum_{i} f_{i}^{2}$, and if $f$ has (total) degree $\leqslant d$, then it is obvious that the $f_{i}$ must have (total) degrees $\leqslant \frac{d}{2}$, since leading terms cannot cancel. The theorem, and its proof, are a generalization of this simple remark.

For the proof of the theorem, we need to use some (easy) ideas from algebraic geometry and from real algebraic geometry. For the first we refer to Hartshorne's book [10], for the second to [3] or [14]. In particular, we need the notion of the real spectrum Sper $A$ of $A$, and how it relates to the semialgebraic subsets of $V(\mathbb{R})$.

Proof. We can assume that $V$ is irreducible. Fix an irreducible component $Z$ of $\bar{V}-V$, then $Z$ has codimension one in $\bar{V}$, and hence defines a discrete valuation $v_{Z}$ of $\mathbb{R}(V)$, the function field of $V$. Namely, $v_{Z}(f)$ is the (vanishing, resp. pole) order of $f$ along $Z$. The residue field of $v_{Z}$ is $\mathbb{R}(Z)$, the function field of $Z$.

The condition in the theorem implies that $Z(\mathbb{R})$ is Zariski dense in $Z$. It is well known that this is equivalent to the condition that the function field $\mathbb{R}(Z)$ of $Z$ is (formally) real. Therefore, the valuation $v_{Z}$ has a real residue field.

The subset $Z(\mathbb{R}) \cap \bar{K}$ of $Z(\mathbb{R})$ is semialgebraic. Therefore, the hypothesis that $Z(\mathbb{R}) \cap \bar{K}$ is Zariski dense in $Z$ means that the associated constructible subset $(Z(\mathbb{R}) \cap \bar{K})^{\sim}$ in the real spectrum of $Z$ contains an element whose support is the generic point of $Z$. This, in turn, means that the constructible set $\tilde{K}$ of Sper $\mathbb{R}[V]$ contains an element $\alpha$ with support (0) which is compatible with the valuation $v_{Z}$.

Let now $Z_{1}, \ldots, Z_{r}$ be the irreducible components of $\bar{V}-V$, and let $v_{Z_{i}}$ be the discrete valuation of $\mathbb{R}(V)$ associated to $Z_{i}$, as above. For $n \geqslant 0$, let the $\mathbb{R}$-subspace $U_{n}$ of $A$ be defined by

$$
U_{n}=\left\{f \in A: v_{Z_{i}}(f) \geqslant-n \text { for } i=1, \ldots, r\right\}
$$


Then $\operatorname{dim}_{\mathbb{R}}\left(U_{n}\right)<\infty$ for each $n$. This is a particular case of [10, Thm. II.5.19], noticing that $U_{n}=\Gamma\left(\bar{V}, \mathcal{O}_{\bar{V}}(n D)\right)$, where $D$ is the Weil divisor $D=\sum_{i} Z_{i}$ on $\bar{V}$. Moreover, $U_{0} \subset U_{1} \subset \cdots \subset \bigcup_{n} U_{n}=A$.

Now assume that $a_{1}, \ldots, a_{m} \in P$ are such that $a_{1}+\cdots+a_{m} \in U_{n}$, for some $n \geqslant 0$. Each $a_{i}$ is positive in $\alpha$. Since $\alpha$ is compatible with $v_{Z_{i}}$, for each $i$, it follows from [19, Lemma 0.2] that $v_{Z_{i}}\left(\sum_{j} a_{j}\right)=\min _{j} v_{Z_{i}}\left(a_{j}\right)$, for each $i$. In particular, each summand $a_{j}$ lies itself in $U_{n}$.

From this observation it is easy to see that the preorder $P$ is stable. Indeed, if $P=$ $\operatorname{PO}\left(f_{1}, \ldots, f_{r}\right)$, and if $a \in A$ and $1 \leqslant j_{1}<\cdots<j_{s} \leqslant r$ are such that $a^{2} f_{j_{1}} \ldots f_{j_{s}} \in U_{n}$, then $v_{Z_{i}}\left(a^{2}\right) \geqslant-n-\sum_{k=1}^{s} v_{Z_{i}}\left(f_{j_{k}}\right)$ for each $i$, and so it is clear that $P \cap U_{n} \subset$ $\Sigma\left(U_{N} ; f_{1}, \ldots, f_{r}\right)$ for sufficiently large $N$. (Explicitly, it suffices to take $2 N \geqslant n+$ $\max _{i} \sum_{j=1}^{r} m_{i j}$, where $m_{i j}=\max \left\{0, v_{Z_{i}}\left(f_{j}\right)\right\}$.)

From the hypotheses, it follows that $K$ is Zariski dense in $V$. Therefore $P$ is closed by Corollary 2.11 .

2.16. Remark. Observe that the hypotheses in the theorem depend only on $V$ and $K$, but not on the particular choice of the preorder $P$. In the next section we will apply the theorem to exhibit classes of basic closed sets which admit no finite presentation by non-strict inequalities which would solve the moment problem.

In the next section, we will apply the following result proved in [19, Prop. 6.1]:

2.17. Proposition. Let $A$ be a f. g. $\mathbb{R}$-algebra, let $V=\operatorname{Spec} A$, and let $P$ be a finitely generated preorder in $A$. Assume that $K=\mathscr{S}(P)$ has (topological) dimension $\geqslant 3$. Then there exists $f \in \mathbb{R}[V]$ with $f \geqslant 0$ on $V(\mathbb{R})$ but $f \notin P$. In particular, $P$ is not saturated.

2.18. Example. Let $P$ be a f. g. preorder in $A$ for which $K=\mathscr{S}(P)$ is compact. If $K$ has dimension $\geqslant 3$, then, by $2.17, P$ is not saturated, and hence is not closed by Schmüdgen's theorem (c.f. 3.2 below). In particular, if $A$ is reduced and $K$ is Zariski dense in $V, P$ cannot be stable, by 2.11 .

If $\operatorname{dim}(K) \leqslant 2$, however, the question of stability for $P$ is less clear, since $P$ may be saturated. We mention a non-trivial example: Let $C$ be a smooth affine curve over $\mathbb{R}$ for which $C(\mathbb{R})$ is compact. Let $P=\Sigma \mathbb{R}[C]^{2}$, the preorder of all sums of squares. It can be shown that $P$ is saturated [20]. In any case, $P$ is stable if, and only if, for each integer $d \geqslant 0$, there is an integer $N(d) \geqslant 0$, such that every sum of squares $f \in \mathbb{R}[C]$ whose pole orders (in the points at infinity) are $\leqslant d$ is a sum of squares of regular functions whose pole orders are $\leqslant N(d)$. We do not know whether this condition always holds. One can show that it does indeed hold if $C$ has genus one, using the approach from $[19, \S 4]$.

\section{Applications to the moment problem}

We use the previous results on preorders to study the questions raised in $\S 1$. First, we can rephrase Haviland's theorem (1.1) in our terminology as follows: 
3.1. Corollary. Suppose $P$ is a preorder in $\mathbb{R}\left[t_{1}, \ldots, t_{n}\right]$ and $K=\mathscr{S}(P)$. Then $P$ solves the moment problem for $K$ if and only if the closure $\bar{P}$ of $P$ is a saturated preorder.

Proof. If $\bar{P}$ is not saturated, then there is a polynomial $f$ with $f \geqslant 0$ on $K$ but $f \notin \bar{P}$. The argument of 1.4 now generalizes: Since $\bar{P}$ is a convex cone, there exists, by HahnBanach separation, a linear form $L: \mathbb{R}\left[t_{1}, \ldots, t_{n}\right] \rightarrow \mathbb{R}$ with $L \geqslant 0$ on $P$, but $L(f)<$ 0 . Obviously, $L$ cannot come from a positive Borel measure on $K$. Conversely, every linear map $L$ with $L \geqslant 0$ on $P$ satisfies $L \geqslant 0$ on $\bar{P}$. So if $\bar{P}$ is saturated, $P$ solves the moment problem for $K$ by Haviland's theorem.

3.2. Example. Let $A$ be a f. g. $\mathbb{R}$-algebra and $P$ a f. g. preorder in $A$ for which $K=$ $\mathscr{S}(P)$ is compact. Then the saturation $\operatorname{Sat}(P)$ of $P$ is equal to the closure $\bar{P}$ of $P$ in $A$. Indeed, $\operatorname{Sat}(P)$ is closed by 2.4. Conversely, for every $f \in \operatorname{Sat}(P)$ and $\varepsilon>0$ one has $f+\varepsilon \in P$, by Schmüdgen's theorem (1.3), and so $f \in \bar{P}$.

3.3. Corollary. Suppose $P$ is a preorder in $\mathbb{R}\left[t_{1}, \ldots, t_{n}\right]$ and $K=\mathscr{S}(P)$. If $P$ is closed but not saturated, then $P$ does not solve the moment problem for $K$.

3.4. Remark. For the question of whether a given preorder solves the moment problem, we can often pass from affine $n$-space to a smaller algebraic variety; and, as we will see, it is in fact useful to do so. The reason is the following.

Let $P$ be a preorder in $A=\mathbb{R}\left[t_{1}, \ldots, t_{n}\right]$, and let $K=\mathscr{S}(P)$. The support of $P$ is the ideal $\operatorname{supp}(P):=P \cap(-P)$ of $A$. Write $B:=A / \operatorname{supp}(P)$, and let $\pi: A \rightarrow B$ be the natural epimorphism. Then $V=\operatorname{Spec}(B)$ is a closed subvariety of $\mathbb{A}^{n}$, and $K$ is contained in $V(\mathbb{R})$. If $P$ is finitely generated, then $K$ is Zariski dense in $V$. This is seen as follows: Let $f \in A$ with $f \equiv 0$ on $K$. By the real Nullstellensatz (e. g. [14, p. 143]), there is an identity $f^{2 m}+a=0$ in $A$, with $m \geqslant 1$ and $a \in P$. Therefore $f^{2 m} \in \operatorname{supp}(P)$, and so the element $\pi(f)$ is nilpotent in $B$. Note however that the $\operatorname{ring} B$ need not be reduced in general.

Let $Q=\pi(P)$, the preorder in $B$ induced by $P$. The linear maps $L: A \rightarrow \mathbb{R}$ with $L \geqslant 0$ on $P$ are precisely those of the form $L=\bar{L} \circ \pi$, where $\bar{L}: B \rightarrow \mathbb{R}$ is a linear map with $\bar{L} \geqslant 0$ on $Q$. Therefore, the closures of $P$ (in $A$ ) and of $Q$ (in $B$ ) are related by $\bar{P}=\pi^{-1}(\bar{Q})$. On the other hand, the respective saturations are obviously related by $\operatorname{Sat}_{A}(P)=\pi^{-1}\left(\operatorname{Sat}_{B}(Q)\right)$. Therefore, $P$ solves the moment problem for $K \Leftrightarrow \bar{P}=$ $\operatorname{Sat}_{A}(P) \Leftrightarrow \pi^{-1}(\bar{Q})=\pi^{-1}\left(\operatorname{Sat}_{B}(Q)\right) \Leftrightarrow \bar{Q}=\operatorname{Sat}_{B}(Q) \Leftrightarrow$ the closure of $Q$ in $B$ is saturated.

Conversely, if $I$ is an ideal in $A=\mathbb{R}\left[t_{1}, \ldots, t_{n}\right]$, if $B=A / I$ and $\pi: A \rightarrow B$ is the canonical map, and if $Q$ is a preorder in $B$, then starting from a system of generators for $Q$ it is easy to obtain a system of generators for the preorder $P:=\pi^{-1}(Q)$ of $A$. Indeed, if $Q$ is generated by elements $\pi\left(f_{\lambda}\right), \lambda \in \Lambda$, with $f_{\lambda} \in A$, then $P$ is generated by the $f_{\lambda}$ together with $\pm a_{1}, \ldots, \pm a_{m}$, where $a_{1}, \ldots, a_{m}$ is a system of generators for the ideal $I$. In particular, $Q$ is finitely generated iff $P$ is finitely generated. Summarizing some of the above discussion, we have:

3.5. Lemma. Let $P$ be a preorder in $A=\mathbb{R}\left[t_{1}, \ldots, t_{n}\right]$, let $K=\mathscr{S}(P)$, and let $Q$ be the preorder induced by $P$ in $B=A / \operatorname{supp}(P)$. Write $V=\operatorname{Spec}(B)$. 
(a) $P$ solves the moment problem for $K\left(\right.$ in $\left.\mathbb{R}^{n}\right)$ if and only if the closure of $Q$ in $B$ is saturated.

(b) $P$ is finitely generated if and only if $Q$ is finitely generated. In either case, the subset $K$ of $V(\mathbb{R})$ is Zariski dense in $V$.

3.6. Corollary. Let $K$ be a basic closed semialgebraic subset of $\mathbb{R}^{n}$. Let $I$ be the ideal of all polynomials in $A=\mathbb{R}\left[t_{1}, \ldots, t_{n}\right]$ which vanish identically on $K$, and put $B=A / I$. The following two conditions are equivalent:

(i) There exists a finite family of polynomials which solves the moment problem for $K$;

(ii) the saturated preorder $\mathscr{P}(K)$ in $B$ contains a dense preorder which is finitely generated.

In view of the lemma and its corollary, Thm. 2.14 allows us to obtain many examples of basic closed semialgebraic sets $K$ for which the moment problem is not solved by any finite family of polynomials.

3.7. Proposition. Let $f_{1}, \ldots, f_{r} \in \mathbb{R}\left[t_{1}, \ldots, t_{n}\right]$ be such that $K=\left\{f_{1} \geqslant 0, \ldots, f_{r} \geqslant 0\right\}$ contains an open cone (i.e., there are $x \in \mathbb{R}^{n}$ and a non-empty open subset $U$ of $\mathbb{R}^{n}$ with $x+\lambda u \in K$ for every $\lambda \geqslant 0$ and $u \in U)$. Then the preorder $P=\operatorname{PO}\left(f_{1}, \ldots, f_{r}\right)$ is stable and closed. If $n \geqslant 2, P$ is not saturated, and hence $f_{1}, \ldots, f_{r}$ do not solve the moment problem for $K$.

Proof. By Thm. 2.14, $P$ is stable and closed, and by Prop. 2.17, $P$ is not saturated if $n \geqslant 3$. Consider the case $n=2$ (c.f. also [19, Rem. 6.7]). We can find a smooth irreducible curve $C$ in the affine plane, of genus $\geqslant 1$, which has exactly one point at infinity and for which the set $K \cap C(\mathbb{R})$ is unbounded. Indeed, after an affine change of coordinates, $K$ contains the positive quadrant, and we can take $C$ to be the curve $t_{2}^{2}=t_{1}\left(t_{1}^{2}+1\right)$, for example.

Let $\pi: \mathbb{R}\left[t_{1}, t_{2}\right] \rightarrow \mathbb{R}[C]$ be the natural (restriction) homomorphism. By $[19$, Cor. 3.9], there exists a psd function $g \in \mathbb{R}[C]$ which is not contained in $\pi(P)$, the preorder induced by $P$ in $\mathbb{R}[C]$. By loc. cit., Thm. 5.6, $g$ can be lifted to a psd polynomial $f \in \mathbb{R}\left[t_{1}, t_{2}\right]$, i.e., $\pi(f)=g$. Clearly $f \notin P$, and in particular, $P$ is not saturated.

3.8. Remarks. 1 . The fact that $P$ is closed is also proven by Kuhlmann and Marshall in [15, Thm. 3.5]. Applying results from [19], they deduce from this that the $f_{i}$ do not solve the moment problem for $n \geqslant 2$, in the same way that we do.

2. In contrast to 3.7 , it is not enough to assume that $K$ contains a cylinder. For example, in $[15,5.1]$, it is shown if $K$ is a cylinder in $\mathbb{R}^{2}$ with compact cross section, then there is a finite set of polynomials which solves the moment problem for $K$.

We recall some terminology: If $C$ is a smooth irreducible affine curve over $\mathbb{R}$, there exists (up to isomorphism) a unique smooth irreducible projective curve $\bar{C}$ over $\mathbb{R}$ which contains $C$ as a Zariski open subset, i.e., for which there is a finite subset $T$ of $\bar{C}$ such that $C \cong \bar{C} \backslash T$. The points in $T$ are called the points at infinity of $C$. They are called real or nonreal according to whether they are $\mathbb{R}$-rational or not. 
We can now give a complete solution to the moment problem for one-dimensional closed semialgebraic sets $K$ contained in a smooth curve $C$. There are two possible situations, depending on properties of $C$ and $K$. We start with the first case (for the remaining case, see 3.12 below):

3.9. Theorem. Let $C$ be a smooth affine curve over $\mathbb{R}$, of genus $g \geqslant 1$, and let $P$ be $a$ f. g. preorder in $\mathbb{R}[C]$. Put $K=\mathscr{S}(P)$. Assume that every point of $C$ at infinity is real, and is contained in the closure of $K($ inside $\bar{C}(\mathbb{R}))$. Then $P$ is closed and stable, but not saturated.

Proof. $P$ is stable and closed by Thm. 2.14. On the other hand, by [19, Thm. 3.5], there exists a psd function $f \in \mathbb{R}[C]$ which is not contained in $P$. In particular, $P$ is not saturated.

In particular, if $C$ is a smooth affine curve over $\mathbb{R}$, of genus $g \geqslant 1$, whose points at infinity are all real, and if $K \subset C(\mathbb{R})$ is a closed semialgebraic set whose closure in $\bar{C}(\mathbb{R})$ contains all points at infinity, then the moment problem for $K$ is not solvable by finitely many polynomial functions.

We can even go further and, using a restriction-extension argument, generalize this last fact considerably. The following corollary was inspired by, and generalizes, [15, Cor. 3.10]:

3.10. Corollary. Let $K$ be a basic closed semialgebraic subset of $\mathbb{R}^{n}$. Assume that there exists a smooth curve $C$ in $\mathbb{A}^{n}$, of genus $\geqslant 1$, all of whose points at infinity are real and are contained in the closure of $K \cap C(\mathbb{R})$ (inside $\mathbb{P}^{n}(\mathbb{R})$ ). Then the moment problem for $K$ is not solvable by finitely many polynomials.

Proof. Let $P$ be a f. g. preorder in $A=\mathbb{R}\left[t_{1}, \ldots, t_{n}\right]$ with $\mathscr{S}(P)=K$. We show that the closure $\bar{P}$ is not saturated. Let $\pi: A \rightarrow \mathbb{R}[C]$ be the natural epimorphism, and let $Q=\pi(P)$, the preorder induced by $P$ on $C$. Then $Q$ is finitely generated and has $\mathscr{S}(Q)=K \cap C(\mathbb{R})$. By [19, Thm. 3.5], there exists $g \in \mathbb{R}[C]$ with $g \geqslant 0$ on $C(\mathbb{R})$ but $g \notin Q$. The latter is witnessed by a linear map $L^{\prime}: \mathbb{R}[C] \rightarrow \mathbb{R}$ with $L^{\prime}(g)<0$ but $L^{\prime} \geqslant 0$ on $Q$. The linear map $L:=L^{\prime} \circ \pi: A \rightarrow \mathbb{R}$ satisfies $L \geqslant 0$ on $P$. By loc. cit., Thm. 5.6, we can find an everywhere non-negative polynomial $f \in A$ with $\pi(f)=g$. In particular, $f \in \operatorname{Sat}(P)$, but $f \notin \bar{P}$ since $L(f)<0$.

On the other hand, there are the following positive results for the moment problem on curves. We state them without proofs here, referring instead to [20].

3.11. Theorem ([20]). Let $C$ be an irreducible smooth affine curve over $\mathbb{R}$, let $f_{1}, \ldots$, $f_{r} \in \mathbb{R}[C]$, and put $K=\left\{f_{1} \geqslant 0, \ldots, f_{r} \geqslant 0\right\} \subset C(\mathbb{R})$. Suppose that the following three conditions are satisfied:

(1) Either $C$ has a nonreal point at infinity, or it has a real point at infinity which does not lie in the closure of $K$ (inside $\bar{C}(\mathbb{R}))$; 
(2) $f_{1} \ldots f_{r}$ has vanishing order $\leqslant 2$ in each point of $K$;

(3) if $M$ is an isolated point of $K$, then $\operatorname{ord}_{M}\left(f_{i}\right) \leqslant 1$ for every $i$.

Then the preorder $\mathrm{PO}\left(f_{1}, \ldots, f_{r}\right)$ in $\mathbb{R}[C]$ is saturated.

3.12. Theorem ([20]). Let $C$ be an irreducible smooth affine curve over $\mathbb{R}$. Let $K$ be a closed semi-algebraic subset of $C(\mathbb{R})$, and let $P=\mathscr{P}(K)$, the saturated preorder in $\mathbb{R}[C]$ associated with $K$. Assume that at least one of the following three conditions is satisfied:

(i) C is rational;

(ii) C has at least one nonreal point at infinity;

(iii) C has a real point at infinity which does not lie in the closure of $K($ in $\bar{C}(\mathbb{R}))$.

Then the following are true:

(a) $P$ is finitely generated. In particular, the moment problem for $K$ can be solved by finitely many functions.

(b) If either (ii) or (iii) holds, then $P$ is in fact generated by two elements, and even by one element if $K$ has no isolated points.

Sketch of proof. In case (i), one can reduce to $C=\mathbb{A}^{1}$, i.e. to the polynomial ring $\mathbb{R}[t]$. In this case the proof becomes elementary, see [15, Thm. 2.2], for example. For the remaining cases one has, by Thm. 3.11, to show that one can find polynomial functions $f_{1}, \ldots, f_{r} \in \mathbb{R}[C]$ such that $K=\left\{M \in C(\mathbb{R}): f_{1}(M) \geqslant 0, \ldots, f_{r}(M) \geqslant 0\right\}$, and such that the $f_{i}$ satisfy conditions (2) and (3) from 3.11; moreover, that this is even possible with $r=2$ or $r=1$, respectively. This can be achieved using methods similar to those used in $[19, \S 2]$. For details, we refer to [20].

3.13. Remark. Observe that the condition that at least one of (i)-(iii) holds in 3.12 is the precise complement of the condition in 3.9. Therefore, taking together 3.9 and 3.12 , we have obtained a complete answer to the question whether the moment problem for $K$ is solvable by finitely many polynomials, in the case when $K$ is contained in a smooth curve.

3.14. Example. We illustrate our results on curves by a series of examples.

1. Let $C$ be an irreducible smooth affine curve over $\mathbb{R}$, embedded into $\mathbb{A}^{n}$ as a Zariski closed subset, for some $n$. Let $I=\left(g_{1}, \ldots, g_{m}\right)$ be the vanishing ideal of $C$ inside $\mathbb{R}\left[t_{1}, \ldots, t_{n}\right]$, and let $K$ be a closed semialgebraic subset of $C(\mathbb{R})$. Assume that $C$ and $K$ satisfy (ii) or (iii) from 3.12. Then the moment problem for $K$ (considered as a closed subset of $\mathbb{R}^{n}$ ) can be solved by $2 m+2$ polynomials, and even by $2 m+1$ if $K$ does not contain an isolated point. Namely, it suffices to take two polynomials whose restrictions to $C$ generate $P=\mathscr{P}(K)$ in $\mathbb{R}[C]$, together with $\pm g_{1}, \ldots, \pm g_{m}$. If $K=C(\mathbb{R})$ (so $C$ has a nonreal point at infinity), the moment problem for $K$ is solved by $\pm g_{1}, \ldots, \pm g_{m}$ alone. Both assertions follow from 3.12. 
2. For an explicit example, let $C$ be the plane affine curve with equation $f(x, y)=x^{3}+y^{3}+1=0$. Then $C$ is smooth, of genus one, and has 3 geometric points at infinity, of which one is real and the other two are complex conjugate. According to the remark before, the moment problem can be solved by $\leqslant 4$ polynomials for any closed semialgebraic subset $K$ of $C(\mathbb{R}) \subset \mathbb{R}^{2}$. Using the criterion from 3.11, it is easy to verify, for example, that the moment problem for $K_{1}=$ $C(\mathbb{R}) \cap\{x \geqslant 0\}$ is solved by $x, f,-f$, that the moment problem for $K_{1} \cup\{(-1,0)\}$ is solved by $x+y+1,-y, \pm f$, and that the moment problem for $K=C(\mathbb{R})$ is solved by $\pm f$.

3. Let $C$ be the plane hyperbola with equation $y^{2}=x^{2}+1$. The moment problem for the set $C(\mathbb{R}) \subset \mathbb{R}^{2}$ is solved by $\pm\left(y^{2}-x^{2}-1\right)$. The moment problem for $C(\mathbb{R}) \cap\{x \geqslant 0, y \geqslant 0\}$ is solved by $x+y-1, \pm\left(y^{2}-x^{2}-1\right)$; and so on.

4. Let $q(x) \in \mathbb{R}[x]$ be a square-free polynomial, and let $K$ be a closed semialgebraic subset of $C(\mathbb{R}):=\left\{(x, y) \in \mathbb{R}^{2}: y^{2}=q(x)\right\}$. If $\operatorname{deg}(q) \leqslant 2$, then the moment problem for $K$ can be solved by finitely many polynomials, since the curve $y^{2}=q(x)$ is rational. If $\operatorname{deg}(q) \geqslant 3$ and $K$ is not compact, the moment problem for $K$ cannot be solved by finitely many polynomials (3.9). In the case $K=C(\mathbb{R})$, this is also shown directly in $[15,3.7]$.

5. Generalizing part of the last example, let $C$ be an irreducible smooth plane curve with equation $x^{n}+f(x, y)=0$, where every monomial in $f(x, y)$ has total degree $\leqslant n-1$. If $n \geqslant 3$, then the moment problem cannot be solved by finitely many polynomials for any non-compact closed semialgebraic subset of $C(\mathbb{R})$ (in particular, for $C(\mathbb{R})$ itself, since $C(\mathbb{R})$ is not compact). Indeed, $C$ has precisely one point at infinity, and this point is real; moreover, $C$ is not rational. So the assertion follows from 3.9.

6. We remark that our questions from the introduction are completely settled for (closed semialgebraic) subsets $K$ of $\mathbb{R}$, in [15, §2].

Finally, we can settle some of the open problems raised in [15]. Open Problem 4 asks for an irreducible smooth affine curve over $\mathbb{R}$ for which the sums of squares are not closed. Such a curve cannot exist:

3.15. Corollary. If $C$ is an irreducible smooth affine curve over $\mathbb{R}$, then the preorder $\Sigma \mathbb{R}[C]^{2}$ of all sums of squares is closed in $\mathbb{R}[C]$.

Proof. If $C$ has a nonreal point at infinity, then $\Sigma \mathbb{R}[C]^{2}$ is even saturated ([20]; if $C$ is not rational, this is also contained in Thm. 3.11 as a special case; if $C$ is rational, it is clear anyway). If all points of $C$ at infinity are real, 3.9 applies.

We can also answer Open Problems 6 and 7 from [15]. Namely, the preorders $P_{1}:=\mathrm{PO}(x, 1-x)$ and $P_{2}:=\mathrm{PO}(1+x, 1-x, 1+y, 1-y)$ in $\mathbb{R}[x, y]$ are both saturated, and in particular, are closed. This is proved in [20]. Problem 7 asked whether $P_{2}$ is closed. Problem 6 asked whether for every $f \in \operatorname{Sat}\left(P_{1}\right)$ it is true that $f+\varepsilon \in P_{1}$ for all $\varepsilon>0$. 


\section{References}

[1] C. Berg, J. Christensen, C. Jensen, A remark on the multidimensional moment problem. Math. Ann. 243 (1979), 163-169. Zbl 416.46003

[2] C. Berg, P. H. Maserick, Polynomially positive definite sequences. Math. Ann. 259 (1982), 487-495. Zbl 486.44004

[3] J. Bochnak, M. Coste, M.-F. Roy, Real Algebraic Geometry. Ergeb. Math. Grenzgeb. (3) 36, Springer 1998. Zbl 912.14023

[4] N. Bourbaki: Espaces vectoriels topologiques, Chapitres 1 à 5. Masson 1981. Zbl 482.46001

[5] N. Bourbaki: Topologie Générale, Chapitres 1 et 2. Hermann 1965. Zbl 249.54001

[6] G. Cassier, Problème des moments sur un compact de $\mathbb{R}^{n}$ et dècompositions de polynômes a plusiers variables. J. Funct. Anal. 58 (1984), 254-266. Zbl 556.44006

[7] M. D. Choi, T. Y. Lam, B. Reznick, Sums of squares of real polynomials. In: K-Theory and Algebraic Geometry: Connections with Quadratic Forms and Division Algebras (B. Jacob, A. Rosenberg, eds.), Proc. Symp. Pure Math. 58.2, 103-126. Amer. Math. Soc. 1995. Zbl 821.11028

[8] A. Devinatz, Two parameter moment problems. Duke Math. J. 24 (1957), 481-498. Zbl 081.10104

[9] H. Hamburger, Über eine Erweiterung des Stieltjesschen Momentproblems, Parts I, II, III. Math. Ann. 81 (1920), 235-319, 82 (1921), 20-164, 168-187.

[10] R. Hartshorne, Algebraic Geometry. Springer 1977. Zbl 367.14001

[11] F. Hausdorff, Summationsmethoden und Momentfolgen I. Math. Z. 9 (1921), 74-109.

[12] E. K. Haviland, On the momentum problem for distribution functions in more than one dimension II. Amer. J. Math. 58 (1936), 164-168. Zbl 015.10901

[13] D. Hilbert, Über die Darstellung definiter Formen als Summe von Formenquadraten. Math. Ann. 32 (1888), 342-350.

[14] M. Knebusch, C. Scheiderer, Einführung in die reelle Algebra. Vieweg 1989. Zbl 732.12001

[15] S. Kuhlmann, M. Marshall, Positivity, sums of squares and the multi-dimensional moment problem. Preprint 2000.

[16] J. L. McGregor, Solvability criteria for certain $N$-dimensional moment problems. $J$. Approx. Theory 30 (1980), 315-333. Zbl 458.41025

[17] T. Motzkin, The arithmetic-geometric inequalities. In: Inequalities (O. Shisha, ed.), Proc. Symp. Wright-Patterson AFB, August 19-27, 1965, 205-224. Academic Press 1967. Zbl 178.00102

[18] B. Reznick, Concrete aspects of Hilbert's 17th Problem. In: Real Algebraic Geometry and Ordered Structures (C. Delzell, J. J. Madden, eds.), 251-272. Contemp. Math. 253, Amer. Math. Soc. 2000.

[19] C. Scheiderer, Sums of squares of regular functions on real algebraic varieties. Trans. Amer. Math. Soc. 352 (2000), 1039-1069. Zbl 941.14024

[20] C. Scheiderer, Sums of squares in coordinate rings of compact real varieties. Work in progress.

[21] K. Schmüdgen, An example of a positive polynomial which is not a sum of squares of polynomials. A positive, but not strongly positive functional. Math. Nachr. 88 (1979), 385-390. Zbl 424.46041

[22] K. Schmüdgen, Unbounded operator algebras and representation theory. Oper. Theory Adv. Appl. 37, Birkhäuser 1990. Zbl 697.47048 
[23] K. Schmüdgen, The $K$-moment problem for compact semi-algebraic sets. Math. Ann. 289 (1991), 203-206. Zbl 744.44008

[24] J. A. Shohat, J. D. Tamarkin, The Problem of Moments. Math. Surveys 1, Amer. Math. Soc. 1943. Zbl 063.06973

[25] T. J. Stieltjes. Recherches sur les fractions continues. Ann. Fac. Sci. Toulouse (1895), A5A47. Reprinted in Ann. Fac. Sci. Toulouse Math. 4 (1995), no. 4, A5-A47. Zbl 861.01037

Received 19 October, 2000.

V. Powers, Department of Mathematics and Computer Science, Emory University, Atlanta, GA 30322, USA

E-mail: vicki@mathcs.emory.edu

C. Scheiderer, Fakultät für Mathematik, Universität Duisburg, 47048 Duisburg, Germany E-mail: claus@uni-duisburg.de 